\title{
Uterine Corpus Apoplectic Leiomyoma
}

National Cancer Institute

\section{Source}

National Cancer Institute. Uterine Corpus Apoplectic Leiomyoma. NCI Thesaurus. Code C40165.

A morphologic variant of uterine corpus leiomyoma characterized by zones of hemorrhagic infarction surrounded by hypercellular areas. It usually develops in women of childbearing years, particularly those that are pregnant, post-partum, or taking oral contraceptives. 